\title{
Uso de redes sociais, influência da mídia e insatisfação com a imagem corporal de adolescentes brasileiras
}

\author{
Social media consume, media influence and body dissatisfaction \\ among Brazilian female adolescents \\ Ariana Galhardi Lira', Aline de Piano Ganen', Aline Sinhorini Lodi², Marle dos Santos Alvarenga ${ }^{3}$
}

\section{RESUMO}

Objetivo: Avaliar relações entre a influência da mídia e o uso de redes sociais na imagem corporal (IC) de adolescentes do sexo feminino. Métodos: Estudo transversal realizado com meninas adolescentes estudantes de escola pública e de uma organização não governamental da capital e do interior de São Paulo. Além de variáveis sociodemográficas e antropométricas, a avaliação da (IC) foi realizada pela Escala de Silhuetas Brasileiras. A influência da mídia foi avaliada pela subescala 1 de internalização geral da Escala de Atitudes Socioculturais em Relação à Aparência (SATAQ-3). Por fim, foram aplicadas perguntas sobre a frequência de acesso às mídias sociais e possível influência delas na IC. Resultados: Participaram 212 meninas (14,8; DP 1,69 anos), sendo a maioria eutrófica $(65,1 \%)$, pertencentes às classes sociais D e E, com escolaridade materna correspondente ao ensino médio completo; 85,8\% estavam insatisfeitas com a IC; a maioria desejava uma silhueta menor. As meninas que escolheram figuras menores como desejadas apresentaram valores superiores na SATAQ-3 ( $p<0,001$ ). O acesso diário maior de 10 vezes ao dia ao Facebook e Instagram aumentou a chance de insatisfação em 6,57 e 4,47 vezes, respectivamente. Conclusão: As mídias, incluindo as redes sociais, estão associadas à insatisfação da IC de meninas adolescentes.

\section{ABSTRACT}

Objective: To evaluate relationship between media influence and use of social networks in the body image (Bl) of female teenagers. Methods: Transversal study involving students of public school and non-governmental organization in Sao Paulo State, Brazil. Besides the social-demographic variables, the teenagers were evaluated about anthropometric measurements. For the evaluation of the BI it was used the Brazilian Scale of Silhouettes, and for the influence of the media it was used the subscale 1 of The Sociocultural Attitudes Towards Appearance Scale (SATAQ-3). Questions about the social media frequency of access were applied to measure the influence of these medias. Results: 212 females (14.8; DP 1.69 years) participate in the study, $65.1 \%$ normal weight range, belonging $D$ and $E$ social class, most of mother's been high school graduated; $85.8 \%$ were unsatisfied with Bl and the majority desires

\section{Keywords}

Social media, body image, adolescents, social networking. a smaller silhouette. Those that chose smaller figures had higher values in the SATAQ-3 ( $p<$ 0.001). The daily access (more than 10 times) to Facebook and Instagram increased the chance to be Bl unsatisfied 6.57 and 4.47 respectively. Conclusion: The media - including the social networks - is associated with BI dissatisfaction among female teenagers in our sample.

1 Centro Universitário São Camilo. 2 Universidade Federal de São Paulo (Unifesp).

Recebido em

22/5/2017

Aprovado em

3 Faculdade de Saúde Pública - Universidade de São Paulo (USP).

DOI: 10.1590/0047-2085000000166

Endereço para correspondência: Ariana Galhardi Lira

Rua Raul Pompéia, 144 - Vila Pompeia

05008-010 - São Paulo, SP, Brasil

Telefone: (11) 3465-2600

E-mail: arianagalhardi@yahoo.com.br 


\section{INTRODUÇÃO}

O uso de redes sociais e sua influência são um fenômeno relativamente recente e alvo de estudos de várias áreas do conhecimento para compreender os efeitos à sua exposição em diferentes populações ${ }^{1-3}$. Essas novas mídias reforçam o narcisismo e os padrões de beleza vigentes e alguns estudos avaliaram seu impacto sobre a imagem corporal (IC $)^{4,5}$.

A IC pode ser definida como a imagem do corpo construída em nossa mente e os sentimentos, pensamentos e ações em relação ao corpo ${ }^{6}$. A insatisfação corporal é um distúrbio atitudinal da IC, descrito como a avaliação subjetiva negativa da IC, que pode ser avaliada pela discrepância entre a IC real e a idealizada ${ }^{7,8}$. Acredita-se que a internalização do padrão do corpo "ideal", ou seja, a incorporação do valor ao ponto de modificar as atitudes e comportamentos pessoais, é um importante mediador da insatisfação corporal ${ }^{9}$.

A IC é, portanto, influenciada por diversos fatores, e três deles têm maior importância: os pais, os amigos e a mídia ${ }^{10}$. Esta última, sinônimo de "meios de comunicação social", é a mais pervasiva das influências ${ }^{10,11}$.

As modificações ocorridas na adolescência, tanto biológica como emocional, podem ser difíceis de lidar, como, por exemplo, o aumento de gordura corporal nas meninas no período pré-menarca e a perda do corpo e do papel e identidade infanti ${ }^{12}$. Os adolescentes, especialmente as meninas, tendem a apresentar preocupações com o peso corporal por desejarem um corpo magro e pelo receio de rejeição, constituindo um grupo mais vulnerável às influências socioculturais e à mídia ${ }^{13}$. Além disso, são importantes consumidores de tendências, entre elas, usam intensamente as mídias sociais como modo de comunicação e "informação", e estas, por sua vez, parecem exercer importante influência sobre a insatisfação corporal.

Holland e Tiggemann ${ }^{14}$, em revisão sistemática sobre o impacto do uso das mídias sociais na IC e no comer transtornado, apontaram como problemática algumas atividades nessas redes, tais como visualização e upload de fotos. Essas atividades favoreceram a comparação social baseada na aparência, reforçando sua relação com a IC e o comer transtornado.

Entretanto, poucos estudos foram feitos a esse respeito, e nenhum deles foi realizado no Brasil e poucos foram com adolescentes ${ }^{3,5,15}$. Nesse contexto, o objetivo deste estudo foi avaliar as relações entre a influência da mídia, o uso de redes sociais e a insatisfação com a IC de meninas adolescentes.

\section{MÉTODOS}

Trata-se de um estudo transversal, cuja amostra foi composta por adolescentes do sexo feminino, em duas escolas públicas - uma em São Paulo capital e outra em Marília, in- terior do estado de São Paulo - e em uma organização não governamental (ONG) na capital. Como critérios de inclusão, adotou-se idade entre 10 e 19 anos, e estar matriculada nas escolas e na ONG. Os critérios de não inclusão foram algum tipo de condição ou síndrome que comprometesse o desempenho cognitivo, devido às possíveis dificuldades em responder adequadamente aos questionários, bem como estar grávida no momento da coleta.

Os procedimentos para o desenvolvimento da pesquisa respeitaram os preceitos estabelecidos pelo Conselho Nacional de Saúde. As escolas assinaram termo de coparticipação, os pais, o termo de consentimento livre e esclarecido (TCLE) e as adolescentes, o termo de assentimento livre e esclarecido (TALE). O estudo foi aprovado pelo Comitê de Ética do Centro Universitário São Camilo (COEP) sob o CAAE: 60801616.5.0000.0062.

A coleta de dados ocorreu em dezembro 2016 na ONG e em fevereiro de 2017 nas escolas. Os pais receberam, juntamente com o TCLE, questões de renda familiar e escolaridade. A renda familiar foi analisada em faixas de salários mínimos (SM) nas categorias: menos de 2 SM, de 2 a 4 SM, de 4 a 10 SM, de 10 a 20 SM e mais 20 SM, que corresponde respectivamente às classes sociais $(E, D, C, B, A)^{16}$. A escolaridade materna foi classificada nas categorias ensino fundamental incompleto ou completo, ensino médio incompleto ou completo, ensino superior incompleto ou completo.

As adolescentes tiveram peso e estatura aferidos seguindo a metodologia descrita pelo Sistema de Vigilância Alimentar Nutricional (Sisvan) para o cálculo do índice de massa corporal (IMC). Para a caracterização do estado nutricional (EN), utilizou-se o escore z, classificado, por meio do software AnthroPlus, em magreza acentuada, magreza, eutrofia, sobrepeso, obesidade e obesidade grave, segundo critérios da Organização Mundial de Saúde ${ }^{17,18}$.

As adolescentes receberam os questionários da pesquisa para preenchimento em casa. Para avaliar a satisfação com a IC, foi aplicada a Escala de Silhuetas Brasileiras validada para adolescentes ${ }^{19}$, composta por figuras de silhuetas numeradas de 1 a 15, para as quais as médias de IMC correspondentes variam de 12,5 a 47,5 kg/m², com diferença constante de 2,5 pontos $^{20}$. Elas deveriam responder "a figura que melhor representa seu tamanho atual" e "a figura que você gostaria de ter". A insatisfação foi avaliada por meio da discrepância entre a figura "desejada" e a figura "atual". Foram consideradas satisfeitas as adolescentes que tiveram escore igual a 0. Resultados superior ou igual a +1 e inferior ou igual a -1 foram considerados insatisfeitos ${ }^{20}$. A classificação foi realizada com três possíveis desfechos: satisfeita, desejo de silhueta menor e desejo de silhueta maior.

A influência da mídia foi avaliada pela subescala 1 (internalização geral dos padrões de beleza) da Escala de Atitudes Socioculturais em Relação à Aparência (SATAQ-3) ${ }^{21}$. Essa 
escala investiga as crenças e pensamentos sobre o corpo e verifica a aceitação dos padrões de aparência socialmente estabelecidos. As questões são pontuadas numa escala do tipo Likert, e a subescala 1 tem escore variando entre 9 e 45 pontos; quanto maior o escore, maior a aceitação das mensagens da mídia em relação a ideais estéticos não realistas.

Para avaliação da frequência do uso das redes sociais (Facebook, Instagram, Twitter e Snapchat) e da influência dessas mídias, foram desenvolvidas 13 questões - uma vez que não existem instrumentos específicos para tal avaliação até onde se sabe. Dessas, nove com respostas do tipo Likert (Quadro 1), duas questões abertas (sobre o benefício ou malefício de seguir as redes sociais) e uma questão sobre quais redes sociais elas possuíam e a outra sobre a frequência de acessos.

As análises estatísticas foram realizadas com uso do software Statistical Package for the Social Sciences (SPSS) versão 20.0; o nível de significância adotado foi de $p<0,05$. A distribuição dos dados foi verificada por meio do teste de Kolmogorov-Smirnov. Análises descritivas foram detalhadas por meio de números absolutos e proporções para dados categóricos. Já os dados quantitativos foram apresentados pela média, desvio-padrão, mediana e moda.

Para a comparação entre as médias da SATAQ-3 (dados paramétricos), utilizou-se o teste t de Student para amostras independentes e Anova com um único fator seguido do post-hoc de Bonferroni.

A análise de satisfação corporal foi realizada pela equação (figura atual - figura desejada) com os possíveis desfechos de satisfação ou insatisfação. Análise entre a satisfação corporal (satisfeita versus insatisfeita) e o uso das redes sociais foi realizada por regressão logística (risco de pertencer ao grupo "insatisfeita") univariada. A análise de regressão binária foi utilizada para estimar os valores de chance (odds ratio - OR) e respectivos intervalos de $95 \%$ de confiança (IC 95\%), considerando insatisfeita como desfecho. Foi utilizado o método Enter como forma de definir o modelo final. Os modelos de regressão (logística) foram ajustados para idade, estado nutricional, classe social e escolaridade materna. Os modelos finais foram compostos de variáveis com $p<0,20$ na análise ajustada.

A análise das perguntas sobre mídias sociais foi realizada atribuindo-se um escore, por meio da somatória das respostas das questões do Quadro 1, com a pontuação: $0=$ nunca, $1=$ raramente, 2 = às vezes, $3=$ frequentemente e $4=$ sempre. Esse escore foi correlacionado com o da SATAQ-3 e o escore da insatisfação corporal (atual - desejado), por meio do teste de Pearson e Spearman, respectivamente.

\section{RESULTADOS}

Foram convidadas para participar do estudo 594 adolescentes: 454 residentes em São Paulo capital e 140 em Marília;
243 (40,9\%) não tiveram interesse em participar, 12 (2\%) não tinham redes sociais e $127(21,4 \%)$ não devolveram os questionários - perda de 64,3\%. Dessa forma, o total de participantes foi de 212 meninas com idades entre 10 e 18 anos (média 14,8; DP 1,69).

Embora a amostragem seja não probabilística (por conveniência), um cálculo do poder do $\mathrm{N}$ amostral foi realizado pelo software GPower 3.1.7, com a opção a posteriori para uso de regressão logística univariada. No caso do $\mathrm{N}$ amostral desta pesquisa ( $n=212$ ), com nível de significância adotado de $p<0,05$, tem-se que o poder observado da análise é de $94 \%$.

A amostra foi composta em sua maioria, por adolescentes com idade entre 15 e 19 anos, eutróficas, pertencentes às classes sociais $D$ e E, e com escolaridade materna correspondente ao ensino médio completo.

A análise da satisfação corporal com relação ao EN evidenciou maior insatisfação para aquelas com sobrepeso e obesidade, mas a maioria das eutróficas também estava insatisfeita (Tabela 1). Não houve diferença na satisfação da IC no que se refere à classe social e escolaridade materna (dados não demonstrados).

Tabela 1. Frequência de satisfação com a imagem corporal de acordo com a idade, estado nutricional de adolescentes do sexo feminino - São Paulo, 2017

\begin{tabular}{lccc}
\hline Variáveis & $\begin{array}{c}\text { Satisfeita } \\
(\mathrm{f} \mathrm{e} \%)\end{array}$ & $\begin{array}{c}\text { Insatisfeita } \\
(\mathrm{f} \mathrm{e} \%)\end{array}$ & p-valor $^{1}$ \\
\hline & $30(14,2)$ & $182(85,8)$ & \\
$\begin{array}{l}\text { Idade (anos) } \\
10-14\end{array}$ & $10(12,2)$ & $72(87,8)$ & 0,516 \\
$15-19$ & $20(15,4)$ & $110(84,6)$ & \\
Estado nutricional & & & \\
Magreza & --- & --- & 0,022 \\
Eutrofia & $27(19,6)$ & $111(80,4)$ & \\
Sobrepeso & $2(3,8)$ & $51(96,2)$ & \\
Obesidade & $1(6,2)$ & $15(93,8)$ & \\
Obesidade grave & $0(0)$ & $5(100)$ & \\
\hline
\end{tabular}

: $p<0,05$, teste qui-quadrado.

A análise da influência da mídia, por meio da subescala 1 da SATAQ-3, encontrou média de 24,78 pontos (DP 9,51) para a amostra total, cuja distribuição foi de normalidade ( $p$ $=0,390$ ). Os escores médios na subescala 1 do SATAQ-3 foram analisados com relação a idade, EN, dados sociodemográficos e insatisfação com a IC (Tabela 2), e não houve diferença no que se refere a classe social e escolaridade materna (dados não demonstrados).

Encontrou-se diferença nas médias da subescala 1 da SATAQ-3 de acordo com a satisfação corporal: meninas que escolheram figuras menores como desejadas apresentaram valores superiores comparados às satisfeitas com IC. 
Tabela 2. Escores médios da subescala 1 da Escala de Atitudes Socioculturais em Relação à Aparência (SATAQ-3) de acordo com idade, estado nutricional e satisfação com a imagem corporal de adolescentes do sexo feminino - São Paulo, 2017

\begin{tabular}{lll}
\hline \multirow{2}{*}{ Variáveis } & \multicolumn{2}{c}{ Subescala 1 SATAQ } \\
\cline { 2 - 3 } & Média & p-valor ${ }^{1}$ \\
\hline
\end{tabular}

Idade (anos)

$10-14$

15-19

$23,98(9,20)$

0,568

Estado nutricional

Magreza

Eutrofia

$---$

Sobrepeso

$23,78(8,93)$

Obesidade

$27,85(10,65)$

Obesidade grave

$23,31(8,92)$

$24,40(10,21)$

Satisfação com a imagem corporal

\begin{tabular}{lr} 
Satisfeita & $18,17^{*}(7,36) \quad<0,001$ \\
Insatisfeita (desejo de silhueta menor) & $26,46(9,95)$ \\
Insatisfeita (desejo de silhueta maior) & $24,51(7,90)$ \\
\hline
\end{tabular}

1: $p<0,05$ diferença significativa entre os grupos.

* Comparação entre satisfeita vs. desejo de silhueta menor.

Uma análise de regressão logística univariada foi realizada utilizando a frequência de acesso às redes sociais com as opções mensal, semanal, de 1 a 5 vezes por dia, de 5 a 10 vezes por dia, mais de 10 vezes por dia e resultado na insatisfação corporal (Tabela 3).

Observou-se, na análise bruta, que aquelas que acessavam as redes sociais Facebook e Instagram diariamente e acessavam o Snapchat de 1 a 5 vezes e de 5 a 10 vezes por dia tinham maior chance de serem insatisfeitas com sua IC, comparadas àquelas que acessavam mensalmente. Nessa mesma análise, ressalta-se que o acesso diário maior que 10 vezes ao Facebook e Instagram aumentaram a chance de ser insatisfeita com a IC em 6,57 e 4,47 vezes, respectivamente, quando comparadas àquelas que acessavam mensalmente.

A partir da análise ajustada, observa-se que, independentemente da idade, EN, classe social e escolaridade materna, aquelas que acessavam o Facebook de 1 a 5 vezes e de 5 a 10 vezes por dia tiveram 4,1 e 4,7, respectivamente, mais chances de serem insatisfeitas com sua IC $(p<0,081)$. Nessa mesma análise, verificou-se que as adolescentes que acessam o Instagram mais de 10 vezes ao dia - independentemente dessas variáveis - apresentaram 4,1 vezes mais chances de serem insatisfeitas com a IC $(p<0,05)$.

Para 75,9\% ( $N=161)$, havia benefícios em seguir as redes sociais, sendo os mais frequentemente citados: estar informada, se comunicar com amigos e pessoas que estão longe, conhecer novas pessoas, ter acesso a informações sobre dieta e exercício e divertir-se. Para $60 \%(N=127)$, não havia nenhum malefício em seguir essas redes; daquelas que dis-
Tabela 3. Relação entre a insatisfação com a imagem corporal e a frequência de uso das redes sociais por adolescentes do sexo feminino ( $N=212)$ - São Paulo, 2017

\begin{tabular}{|c|c|c|c|c|}
\hline \multirow{2}{*}{ Redes sociais } & \multicolumn{2}{|c|}{ Insatisfeita (bruta) } & \multicolumn{2}{|c|}{ Insatisfeita (ajustada) } \\
\hline & OR (IC95\%) & $\mathrm{p}$-valor ${ }^{1}$ & OR (IC95\%) & p-valor ${ }^{2}$ \\
\hline Facebook & & $<0,001$ & & 0,081 \\
\hline Mensal & 1 & & 1 & \\
\hline 1-5 diário & $3,33(1,43-4,89)$ & & $4,18(2,43-8,69)$ & \\
\hline 5-10 diário & $3,43(2,52-6,87)$ & & $4,78(4,56-12,87)$ & \\
\hline > 10 diário & $6,57(4,38-8,96)$ & & $7,66(0,84-2,91)$ & \\
\hline Semanal & $4,66(0,78-4,98)$ & & $1,26(0,49-3,27)$ & \\
\hline Instagram & & 0,021 & & 0,029 \\
\hline Mensal & 1 & & 1 & \\
\hline 1-5 diário & $1,85(3,18-6,86)$ & & $1,40(1,05-12,92)$ & \\
\hline 5-10 diário & $3,44(1,52-10,91)$ & & $1,94(1,21-23,65)$ & \\
\hline$>10$ diário & $4,47(2,26-11,81)$ & & $4,11(3,09-10,65)$ & \\
\hline Semanal & $1,23(0,26-5,78)$ & & $0,75(0,23-2,39)$ & \\
\hline Snapchat & & 0,041 & & 0,266 \\
\hline Mensal & 1 & & 1 & \\
\hline 1-5 diário & $2,38(4,46-7,97)$ & & $1,11(0,17-1,33)$ & \\
\hline 5-10 diário & $4,00(2,15-6,80)$ & & $2,80(0,31-24,85)$ & \\
\hline > 10 diário & $2,38(0,46-12,11)$ & & $1,78(0,27-11,51)$ & \\
\hline Semanal & $1,48(0,34-6,44)$ & & $1,09(0,20-5,97)$ & \\
\hline Twitter & & 0,090 & & 0,499 \\
\hline Mensal & 1 & & 1 & \\
\hline 1-5 diário & $1,56(0,15-1,97)$ & & $0,50(0,01-1,89)$ & \\
\hline 5-10 diário & $0,43(0,80-3,96)$ & & $0,97(0,79-3,96)$ & \\
\hline$>10$ diário & $1,10(0,97-4,87)$ & & $1,87(0,90-4,82)$ & \\
\hline Semanal & $0,98(0,76-8,27)$ & & $1,22(0,35-19,73)$ & \\
\hline
\end{tabular}

OR: odds ratio; IC 95\%: intervalo de confiança de $95 \%$.

$p<0,05 ; 1$ regressão logística univariada; 2 regressão logística multivariada ajustada para idade, estado nutricional, classe social e escolaridade materna.

seram haver algum, os mais citados foram: "viciar", absorver informações sobre padrão de beleza e ocorrência de bullying.

A frequência de respostas às questões elencadas sobre o uso de mídias sociais pode ser vista no quadro 1.

Observa-se que grande parte concordou que são necessários "força, foco e fé" para emagrecer (Quadro 1) e que um quarto já se sentiu influenciada a cortar da alimentação comidas "não saudáveis". Em torno de um quarto citou que as redes sociais eram fontes de informação sobre o que é um corpo saudável e influenciavam na percepção e relação com o corpo. Contudo, a maioria afirmou não ter feito dietas ou seguido recomendações feitas pelas redes sociais e que as redes sociais não influenciavam suas escolhas alimentares.

O escore formado pelas respostas sobre a influência das redes sociais mostrou correlação média com o escore da subescala 1 da SATAQ-3 (Pearson, $r=0,42, p<0,05$ ), resultado que atesta que as questões elencadas, de alguma forma, também avaliam a influência da mídia. 
Quadro 1. Frequência de respostas sobre redes sociais em adolescentes do sexo feminino ( $N=212)$ - São Paulo, 2017

\begin{tabular}{|c|c|c|c|}
\hline & Nunca & $\begin{array}{l}\text { Raramente ou } \\
\text { às vezes }\end{array}$ & $\begin{array}{l}\text { Frequentemente } \\
\text { ou sempre }\end{array}$ \\
\hline $\begin{array}{l}\text { Você segue redes sociais que } \\
\text { falam sobre dieta e alimentaçãa } \\
\text { "saudável"? }\end{array}$ & $52,8 \%$ & $35,9 \%$ & $11,3 \%$ \\
\hline $\begin{array}{l}\text { Você segue redes sociais que } \\
\text { falam sobre exercício físico? }\end{array}$ & $55,2 \%$ & $30,2 \%$ & $14,6 \%$ \\
\hline $\begin{array}{l}\text { Você concorda que para } \\
\text { emagrecer é necessário "força, } \\
\text { foco e fé"? }\end{array}$ & $6,1 \%$ & $23,1 \%$ & $70,8 \%$ \\
\hline $\begin{array}{l}\text { Você já se sentiu influenciada } \\
\text { a "cortar" da sua alimentação } \\
\text { comidas "não saudáveis"? }\end{array}$ & $36,5 \%$ & $37,9 \%$ & $25,6 \%$ \\
\hline $\begin{array}{l}\text { As redes sociais são fontes de } \\
\text { informação sobre alimentação e } \\
\text { dieta para você? }\end{array}$ & $31,6 \%$ & $48,6 \%$ & $19,8 \%$ \\
\hline $\begin{array}{l}\text { As redes sociais são fontes de } \\
\text { informação sobre o que é um } \\
\text { corpo saudável para você? }\end{array}$ & $32,1 \%$ & $41,9 \%$ & $26 \%$ \\
\hline $\begin{array}{l}\text { Você já fez alguma dieta ou } \\
\text { outra recomendação sobre } \\
\text { alimentação feita pelas redes } \\
\text { sociais? }\end{array}$ & $74,5 \%$ & $17,5 \%$ & $8 \%$ \\
\hline $\begin{array}{l}\text { As redes sociais influenciam } \\
\text { nas suas escolhas alimentares } \\
\text { diárias? }\end{array}$ & $68,9 \%$ & $23,1 \%$ & $8 \%$ \\
\hline $\begin{array}{l}\text { As redes sociais influenciam na } \\
\text { sua percepção e relação com } \\
\text { seu corpo? }\end{array}$ & $48,1 \%$ & $28,3 \%$ & $23,6 \%$ \\
\hline
\end{tabular}

Já o escore formado pelas respostas sobre a influência das redes mostrou correlação fraca com escore de insatisfação corporal (Spearman, $r=0,25, p<0,05$ ), mas aponta, de certa forma, relação entre uso dessas mídias e a insatisfação corporal.

\section{DISCUSSÃO}

Os resultados sobre insatisfação corporal e sua relação com a influência da mídia encontrados estão de acordo com os da literatura, mas este estudo acrescenta ao entendimento do fenômeno a relação também com o uso de redes sociais por meninas adolescentes.

A maioria, mesmo eutróficas, estava insatisfeita com sua $\mathrm{IC}$, principalmente com desejo de ter uma silhueta menor. Encontrou-se insatisfação corporal em mais de 80\%, independentemente da classe social e da escolaridade materna, com maior frequência entre aquelas com sobrepeso e obesidade. Tal achado está em consonância com os da literatura, que aponta que a insatisfação corporal é a norma geral, especialmente para o sexo feminino ${ }^{22,23}$. Parece haver um "descontentamento normativo", que é uma generalização da insatisfação com o peso e as formas corporais, entre as mulheres ${ }^{24}$. Esse fenômeno já foi documentado inclusive em crianças com idades entre 7 e 10 anos $^{25}$. Além de esses ideais de magreza estarem presentes desde muito cedo na vida das crianças (fase na qual constroem sua IC), é na adolescência que os questionamentos e o não enquadramento nos padrões aprendidos podem surgir de forma mais latente, trazendo insatisfação e sofrimento.

O aumento dessa insatisfação entre adolescentes, bem como a influência do EN, é bem documentado na literatura, corroborando nossos achados. Estudos demonstram que meninas com sobrepeso e obesidade sofrem mais a pressão de se encaixarem em modelos e padrões de beleza que apenas uma pequena parcela da população conseguirá de verdade atingir de forma saudável ${ }^{19,25}$. Estudos apontam para a falta de diversidade de corpos como referencial de beleza, parecendo haver somente um modelo do que é belo e dificuldade em lidar com o diferente. Todos desejam ser iguais, como um pré-requisito para ser aceito, sendo o culto à magreza uma norma social, expondo-se à exclusão quem não adere a esse modelo ${ }^{4,15,26}$. A mídia atua reforçando e popularizando maneiras de se atingir o "corpo ideal". A indústria da beleza cria desejos e reforça imagens, e o corpo é associado à ideia de consumo 27,28 .

O desenvolvimento da IC tem influência das alterações físicas e psíquicas pelo contato com os pais, colegas, escola e as experiências ao longo do desenvolvimento, bem como a influência da mídia ${ }^{10}$. As informações do mundo atual, principalmente as ditadas pelas mídias, na maioria das vezes, não coincidem com as ICS reais dos indivíduos, carregadas por questões de ordem biológica, afetiva e social gerando grande insatisfação ${ }^{29}$. A mídia como fonte de informação rápida sobre beleza, forma física e emagrecimento pode desempenhar, portanto, um papel influenciador importante, destacando as redes sociais.

Sobre a relação da IC com a influência da mídia, encontramos que ela é verdadeira, uma vez que as meninas com desejo de silhuetas menores tiveram maior pontuação na subescala 1 da SATAQ-3, ou seja, maior aceitação das mensagens da mídia em relação a ideais estéticos não realistas. A internalização é definida como processo pelo qual padrões de comportamento externos se tornam internos e passam a orientar o comportamento da pessoa, e parece, portanto, um dos mais importantes fatores ${ }^{10,30}$. De qualquer forma, a SATAQ-3 usa em suas questões "pessoas na TV", "cinema", "filme", "revistas" e "videoclipes", e não considera as mídias sociais, que são um fenômeno mais atual. Desconhecemos instrumentos focados na avaliação da influência das mídias sociais sobre a IC, daí a proposta de se avaliar essa relação. E da mesma forma que foi realizado em estudos internacionais, questões específicas foram elencadas com esse objetivo 15,31,32.

Ao se listarem, portanto, questões sobre mídias sociais (e não as da SATAQ-3), encontrou-se correlação entre as respostas e o escore da subescala 1 da SATAQ-3, apontando 
que essas questões sobre mídias sociais avaliam também de alguma forma a influência da mídia. E da mesma forma que houve relação entre insatisfação corporal e escores na SATAQ-3, houve também o achado de que a frequência do acesso às mídias sociais apresentou relação com a razão de chance das adolescentes serem insatisfeitas.

No cenário nacional, a escala SATAQ-3 foi utilizada poucas vezes especialmente com adolescentes. Dunker et al. ${ }^{33}$ encontraram que houve influência do nível socioeconômico nas pontuações da SATAQ-3 entre adolescentes entre 15 e 18 anos; contudo, a classe social não influenciou nossos resultados. Já o estudo de Fortes et al. ${ }^{34}$ encontrou que $46,4 \%$ das adolescentes avaliadas demonstraram alta internalização do ideal de magreza. E Uchoa et al. ${ }^{35}$ identificaram que adolescentes de diferentes culturas e níveis socioeconômicos são influenciados pelos padrões de beleza estabelecidos pela mídia que podem ser determinantes para aumentar a insatisfação com o tamanho e/ou forma do corpo, levando-os a comportamentos não saudáveis visando alcançar a "beleza ideal".

Seja via SATAQ-3, um instrumento desenvolvido e validado para avaliar a influência da mídia, seja via perguntas elencadas sobre as mídias sociais, houve relação do uso/influência da mídia na insatisfação com a IC. Ressalta-se que a maneira como as pessoas se comunicam, consomem mídia, buscam e recebem informações está mudando. Nesse cenário, as redes sociais merecem especial atenção. No presente estudo o Facebook foi a rede social mais acessada $(97,2 \%)$ - 90\% acessavam diariamente -, seguido pelo Instagram. Os dados encontrados corroboram a Pesquisa Brasileira de Mídias (PBM), que encontrou que o Facebook era a rede mais acessada no Brasil. Entretanto, observou-se que as demais redes sociais também foram acessadas por grande parte e com grande frequência, diferentemente do encontrado na PBM ${ }^{36}$. Esse resultado pode ser explicado pelo fato de que a PBM foi realizada em diferentes regiões do país e com públicos e idades variadas. Acredita-se que entre os adolescentes a popularização e a adesão às novidades tecnológicas é maior e ocorre de forma mais rápida.

É relevante conceituar que estamos falando de uma geração que já nasceu na era digital. Essa geração busca na mídia respostas para questões cotidianas e descontentamentos. Se imagens de corpos "perfeitos" são veiculados e essas pessoas as veem repetidamente, começam a acreditar que é uma versão da realidade, e não alcançar tal ideal é motivo de frustração e insatisfação.

Nas questões elencadas sobre mídias sociais, quase um quarto relatou se sentir influenciado na percepção e relação com seus corpos, e as formas citadas pelas quais isso acontece foram: o quanto ser magra é ser bonita, por meio de imagens de corpos perfeitos, fotos de modelos e de barrigas "seca e trincada". Apesar de menos de um quarto ter perce- bido a influência da mídia em sua IC, nosso estudo evidenciou a relação entre o uso e a frequência do acesso dessas redes sociais e a IC de forma mais incisiva. Esses dados corroboram grande e robusta investigação sobre mídia social, a qual verificou que as meninas que despendiam mais de 20 horas por semana na internet eram mais suscetíveis à insatisfação corporal ${ }^{37}$. De forma mais contundente, a maior parte das adolescentes em nossa pesquisa respondeu concordar frequentemente ou sempre que é necessário ter "força, foco e fé" para emagrecer. Essa frase viralizou na internet e começou a ser usada nas redes com o objetivo de "motivar" as pessoas para as práticas de exercícios e disciplina alimentar. Tal crença pode reforçar que, para se alcançar determinado corpo, só é preciso o "desejar" e "se esforçar", levando à culpa e à frustração aqueles que não conseguem.

Poucos estudos até aqui avaliaram o impacto de redes sociais em adolescentes. Pepin e Endresz ${ }^{2}$ encontraram que jovens ( $N=300$; 18-25 anos) da Austrália que usavam o Facebook (principalmente para manter contato com amigos e familiares) sentiam pressão para perder peso e para mudar sua aparência. Correlações foram encontradas entre Instagram e preocupações com a IC e vigilância do corpo, e entre Facebook e Pinterest e pressão percebida pela mídia. Fardouly et al. ${ }^{3}$ verificaram que mulheres entre 17 e 25 anos que passaram algum tempo no Facebook apresentaram humor mais negativo do que aquelas que passaram algum tempo em um site controle.

Contudo, há divergências, uma vez que Kim e Chock ${ }^{5}$, em estudo de jovens adultos (18-25 anos), sugerem que a exposição simples (tempo gasto em mídias sociais) não estava relacionada a preocupações com IC. No entanto, os comportamentos como a visualização e comentários sobre os perfis de colegas, mediados pela comparação entre as aparências, foram significativamente correlacionados com a busca pela magreza 5 .

Hanna et al. ${ }^{38}$, em discussão sobre os mecanismos envolvidos na relação entre mídia social e insatisfação, verificaram que, para ambos os sexos, o uso do Facebook associou-se à maior comparação social e auto-objetificação, que se relacionava à menor autoestima, pior saúde mental e maior vergonha corporal.

O estudo com adolescentes localizado sobre essa temática é o de Tiggemann e Slater ${ }^{15}$ examinando a relação entre a exposição à internet (especialmente o Facebook) e a preocupação com a IC e internalização geral (usando a SATAQ-3 inclusive), com 1.087 meninas (13-15 anos) na Austrália. Os autores identificaram que o tempo gasto na internet foi significativamente relacionado com a internalização do ideal de magreza, a vigilância corporal e o desejo de emagrecer. Além disso, as meninas que passavam em média 1,5 hora/ dia no Facebook tiveram maior preocupação com a IC do que as não usuárias. Concluiu-se que a internet representou 
um potente meio sociocultural de relevância para a IC das adolescentes. Posteriormente, em estudo longitudinal, esses mesmos autores ${ }^{39}$ confirmaram relação entre mídia social e IC, no qual o número de "amizades" foi um preditor prospectivo no aumento da busca pela magreza.

Há evidências, portanto, do impacto do uso das mídias sociais na IC - mesmo que ainda existam poucos estudos sobre a temática -, especialmente entre adolescentes, público mais vulnerável e que consome e é fortemente influenciado por essa mídia. Os dados, de qualquer forma, corroboram nossos achados, que apontaram que as adolescentes que acessavam Facebook, Instagram e Snapchat diariamente tinham mais chance de ser insatisfeitas com sua IC. E no caso do Facebook e do Instagram, essa insatisfação aumentou com a frequência do acesso. Esse achado é muito relevante, uma vez que as pessoas estão intensamente envolvidas com o uso da internet em seus celulares, tablets e computadores, acessando frequentemente essas mídias.

Além disso, verificou-se que, independentemente da idade, EN, classe social e escolaridade materna, as adolescentes que apresentaram maior influência da mídia (pontuação SATAQ-3) tiveram mais chances de ser insatisfeitas com a IC. Apesar de a mídia por si só não ser o único fator que influência na insatisfação corporal, ela parece ser o principal agente influenciador negativo na IC de jovens e crianças ${ }^{40}$.

Deve-se considerar que este estudo teve um delineamento transversal e não permite, portanto, identificar as relações de causa e efeito - os resultados devem ser interpretados considerando-se essa questão. Este estudo tem entre suas limitações o fato de ter utilizado uma amostra não probabilística. Dessa forma, os resultados também não podem ser extrapolados para todos os adolescentes do Brasil - mesmo considerando-se a globalização do uso e acesso às mídias sociais e padrões de beleza. O uso de questionários de autopreenchimento também é sempre passível de vieses, mas no caso da SATAQ-3 e da Escala de Silhuetas Brasileiras, todos foram validados para adolescentes. Já as questões sobre uso de mídias sociais foram elencadas para este estudo e, portanto, não podem ser comparadas com outras avaliações. De qualquer forma, como nenhum estudo nacional até agora avaliou o impacto do uso de redes sociais sobre a IC, ele serve como uma exploração inicial para investigações maiores e com amostras mais amplas.

\section{CONCLUSÃO}

A mídia (especialmente sua internalização), incluindo as redes sociais, está associada com a insatisfação da IC entre adolescentes do sexo feminino em nossa amostra. Sugere-se que novos estudos sejam realizados para melhor esclarecimento de como as mídias sociais afetam a vida da sociedade em relação aos seus corpos e comportamento alimentar.

\section{CONTRIBUIÇÕES INDIVIDUAIS}

Ariana Galhardi Lira Augusto - Foi responsável pela pesquisa desenvolvida como dissertação do Mestrado Profissional em Nutrição do Nascimento à Adolescência do Centro Universitário São Camilo. Realizou o levantamento bibliográfico e a coleta de dados, e participou da análise e interpretação deles, sendo responsável pela concepção deste artigo.

Aline Sinhorini Lodi - Participou da coleta de dados e auxiliou na tabulação e análise deles.

Aline de Piano Ganen - Foi responsável pela concepção do estudo e participou como orientadora do trabalho de mestrado. Participou efetivamente da análise e interpretação dos dados.

Marle dos Santos Alvarenga - Foi responsável pela concepção do estudo e participou como coorientadora do trabalho de mestrado. Participou efetivamente da análise e interpretação dos dados.

Todos os autores revisaram e aprovaram a redação final deste manuscrito.

\section{REFERÊNCIAS}

1. Vermelho SC, Velho APM, Bertoncello V. Sobre o conceito de redes sociais e seus pesquisadores. Educ Pesqui. 2015;41(4):863-81.

2. Pepin G, Endresz N. Facebook, Instagram, Pinterest and co.: body image and social media. J Eat Disord. 2015;3(Suppl 1):022.

3. Fardouly J, Diedrichs PC, Vartanian LR, Halliwell E. Social comparisons on social media: the impact of Facebook on young women's body image concerns and mood. Body Image. 2015;13:38-45.

4. Tiggemann M, Zaccardo M. Strong is the new skinny: a content analysis of fitspiration images on Instagram. J Health Psychol. 2016;1-9.

5. Kim JW, Chock TM. Body image 2.0: Associations between social grooming on Facebook and body image concerns. Computers in Human Behavior. 2015;48:331-9.

6. Slade PD, What is body image? Behav Res Ther. 1994;32(5):497-502.

7. Sato PM, Timerman F, Fabbri AD, Scagliusi FB, Kotait MS. A imagem corporal nos transtornos alimentares: como o terapeuta nutricional pode contribuir para 0 tratamento. In: Alvarenga MS, Scagliusi FB, Philippi ST. Nutrição e transtornos alimentares: avaliação e tratamento. 1ª ed. São Paulo: Manole; 2010. p. 475-97.

8. Thompson JK, Van Den Berg P. Measuring body image attitudes among adolescents and adults. In: Cash TF, Pruzinsky T, editors. Body image: a handbook of theory, research and clinical practice. New York: The Guilford Press; 2002. p. 142-54.

9. Carvalho PHB. Adaptação e avaliação do modelo teórico de influência dos três fatores de imagem corporal para jovens brasileiros [tese]. Juiz de Fora: Processos Psicossociais em Saúde, Universidade Federal de Juiz de Fora; 2016.

10. Thompson JK, Coovert MD, Stormer SM. Body image, social comparison, and eating disturbance: a covariance structure modeling investigation. Int J Eat Disord. 1999;26(1):43-51.

11. Gomes PBMB. Mídia, imaginário de consumo e educação. Educ Soc. 2001;22(74):191-207.

12. Weinberg C. Por que estou assim? Os momentos difíceis da adolescência. São Paulo: Sá; 2007.

13. Zametkin AJ, Zoon CK, Klein HW, Munson S. Psychiatric aspects of child and adolescent obesity: a review of the past 10 years. J Am Acad Child Adolesc Psychiatry. 2004:43(2):134-50.

14. Holland G, Tiggemann M. A systematic review of the impact of the use of social networking sites on body image and disordered eating outcomes. Body Image. 2016;17:100-10. 
15. Tiggemann M, Slater A. NetGirls: the Internet, Facebook, and body image concern in adolescent girls. Int J Eat Disord. 2013;46(6):630-3.

16. IBGE - Instituto Brasileiro de Geografia e Estatística. Pesquisa de Orçamentos Familiares 2008-2009: Manual do Agente de Pesquisa. Rio de Janeiro; 2008.

17. Ministério da Saúde. Secretaria de Atenção à Saúde. Departamento de Atenção Básica. Orientações para a coleta e análise de dados antropométricos em serviços de saúde: Norma Técnica do Sistema de Vigilância Alimentar e Nutricional - Sisvan. Braślia: Ministério da Saúde; 2011.

18. de Onis M, Onyango AW, Borghi E, Siyam A, Nishida C, Siekmann J. Development of a WHO growth reference for school-aged children and adolescents. Bull World Health Organ. 2007;85(9):660-7.

19. Laus MF, Almeida SS, Murarole MB, Braga-Costa TM. Estudo de validação e fidedignidade de escalas de silhuetas brasileiras em adolescentes. Psic Teor Pesq. 2013;29(4):403-9.

20. Kakeshita IS, Silva AIP, Zanatta DP, Almeida SS. Construção e fidedignidade teste-reteste de escalas de silhuetas brasileiras para adultos e crianças. Psic Teor Pesq. 2009;25(2):263-70.

21. Thompson JK, van den Berg P, Roehrig M, Guarda AS, Heinberg LJ. The sociocultural attitudes towards appearance scale-3 (SATAQ-3): development and validation. Int J Eat Disord. 2004;35(3):293-304

22. Petroski EL, Pelegrini A, Glaner MF. Motivos e prevalência de insatisfação com a imagem corporal em adolescentes. Ciênc Saúde Coletiva. 2012;17(4):1071-7.

23. Fortes $L S$, Conti MA, Almeida SS, Ferreira MEC. Insatisfação corporal em adolescentes: uma investigação longitudinal. Rev Psiquiatr Clín. 2013;40(5):167-71.

24. Rodin J, Silberstein L, Striegel-Moore R. Women and weight: a normative discontent. Nebraska Symposium on Motivation; 1984; University of Nebraska. Lincoln: Psychology and Gender. 1985. p. 267-307.

25. Costa LC, Silva DA, Alvarenga MS, de Vasconcelos FA. Association between body image dissatisfaction and obesity among schoolchildren aged 7-10 years. Physiol Behav. 2016;160:6-11.

26. Scagliusi FB, Lourenço BH. A ditadura da beleza e suas consequências no discurso nutricional. In: Alvarenga M, Scagliusi FB, Philippi ST. Nutrição e transtornos alimentares: avaliação e tratamento. Barueri: Manole; 2012. p. 59-83.

27. Siqueira D, Faria A. Corpo, saúde e beleza: representações sociais nas revistas femininas. Comunicação, Mídia e Consumo. 2008;3(9):171-88.
28. Neto PP, Caponi SNC. A medicalização da beleza. Interface-Comunicação, Saúde, Educação. 2007;11(23):569-84.

29. Costa SMB. 0 corpo e a imagem corporal em adolescentes: um estudo numa escola pública no bairro de Jurujuba-Niterói-RJ [dissertação]. Rio de Janeiro: Universidade Federal Fluminense; 2013.

30. Grabe S, Ward LM, Hyde JS. The role of the media in body image concerns among women: a meta-analysis of experimental and correlational studies. Psychol Bull. 2008;134(3):460-76.

31. Brown Z, Tiggemann M. Attractive celebrity and peer images on Instagram: effect on women's mood and body image. Body Image. 2016;19:37-43.

32. Nguyen H, Paiva AL. Relationships Between Social Media Exposure \& Levels of Body Dissatisfaction. 2016.

33. Dunker KLL, Fernandes CPB, Carreira Filho D. Influência do nível socioeconômico sobre comportamentos de risco para transtornos alimentares em adolescentes. I Bras Psiquiatr. 2009;58(3):156-61.

34. Fortes LS, Meireles JFF, Neves CM, Almeida SS, Ferreira MEC. Autoestima, insatisfação corporal e internalização do ideal de magreza influenciam os comportamentos de risco para transtornos alimentares?. Rev Nutr. 2015;28(3):253-64.

35. Uchoa FNM, Lustosa RP, Rocha MTM, Daniele TMC, Deana NF, Alves N, et al. Media influence and body dissatisfaction in Brazilian adolescents. Biomed Res. 2017;28(6):2445-51.

36. Brasil. Presidência da República. Secretaria de Comunicação Social. Pesquisa brasileira de mídia 2015: hábitos de consumo de mídia pela população brasileira. Braśilia: Secom; 2014.

37. Carter A, Forrest JI, Kaida A. Association Between Internet Use and Body Dissatisfaction Among Young Females: Cross-Sectional Analysis of the Canadian Community Health Survey. J Med Internet Res. 2017;19(2):e39.

38. Hanna E, Ward LM, Seabrook RC, Jerald M, Reed L, Giaccardi S, et al. Contributions of Social Comparison and Self-0bjectification in Mediating Associations Between Facebook Use and Emergent Adults' Psychological Well-Being. Cyberpsychol Behav Soc Netw. 2017;20(3):172-9.

39. Tiggemann M, Slater A. Facebook and body image concern in adolescent girls: A prospective study. Int J Eat Disord. 2017;50(1):80-3.

40. Gondoli DM, Corning AF, Salafia EH, Bucchianeri MM, Fitzsimmons EE. Heterosocial involvement, peer pressure for thinness, and body dissatisfaction among young adolescent girls. Body Image. 2011;8(2):143-8. 